\title{
A Combination of Ivermectin and Doxycycline Possibly Blocks the Viral Entry and Modulate the Innate Immune Response in COVID-19 Patients
}

\author{
Dharmendra Kumar Maurya ${ }^{1,2}$.* \\ ${ }^{1}$ Radiation Biology \& Health Sciences Division, Bhabha Atomic Research Centre, \\ Mumbai 400 085, India \\ ${ }^{2}$ Homi Bhabha National Institute, Anushaktinagar, Mumbai-400094, India
}

\section{*Corresponding author}

Dharmendra Kumar Maurya, Ph.D

Scientific Officer/F

Assistant Professor, Homi Bhabha National Institute

Radiation Biology \& Health Sciences Division

Bhabha Atomic Research Centre

Mumbai 400085, India

Email: dkmaurya@barc.gov.in, dkmauryabarc@gmail.com 


\begin{abstract}
The current outbreak of the corona virus disease 2019 (COVID-19), has affected almost entire world and become pandemic now. Currently, there is neither any FDA approved drugs nor any vaccines available to control it. Very recently in Bangladesh, a group of doctors reported astounding success in treating patients suffering from COVID-19 with two commonly used drugs, Ivermectin and Doxycycline. In the current study we have explored the possible mechanism by which these drugs might have worked for the positive response in the COVID19 patients. To explore the mechanism we have used molecular docking and molecular dynamics simulation approach. Effectiveness of Ivermectin and doxycycline were evaluated against Main Protease (Mpro), Spike (S) protein, Nucleocapsid (N), RNA-dependent RNA polymerase (RdRp, NSP12), ADP Ribose Phosphatase (NSP3), Endoribonuclease (NSP15) and methyltransferase (NSP10-NSP16 complex) of SARS-CoV-2 as well as human angiotensin converting enzyme 2 (ACE2) receptor. Our study shows that both Ivermectin and doxycycline have significantly bind with SARS-CoV-2 proteins but Ivermectin was better binding than doxycycline. Ivermectin showed a perfect binding site to the Spike-RBD and ACE2 interacting region indicating that it might be interfering in the interaction of spike with ACE2 and preventing the viral entry in to the host cells. Ivermectin also exhibited significant binding affinity with different SARS-CoV-2 structural and non-structural proteins (NSPs) which have diverse functions in virus life cycle. Significant binding of Ivermectin with RdRp indicate its role in the inhibition of the viral replication and ultimately impeding the multiplication of the virus. Ivermectin also possess significant binding affinity with NSP3, NSP10, NSP15 and NSP16 which helps virus in escaping from host immune system. Molecular dynamics simulation study shows that binding of the Ivermectin with Mpro, Spike, NSP3, NSP16 and ACE2 was quiet stable. Thus, our docking and simulation studies reveal that combination of Ivermectin and doxycycline might be executing the effect by inhibition of viral entry and enhance viral load clearance by targeting various viral functional proteins.
\end{abstract}

Keywords: COVID-19; SARS-CoV-2; Ivermectin; Doxycycline; RNA dependent RNA polymerase; Molecular docking; Molecular dynamics simulation

\title{
Introduction
}

The current outbreak of the corona virus disease 2019 (COVID-19), has affected almost entire World and become pandemic. Because of its high rate of transmission and infectivity, World Health Organization (WHO) has declared it as a global disaster. Different therapeutics are currently being evaluated in clinical trials but there is no specific drug or vaccine that has been proven its effectiveness to date. Most of the current treatment options against COVID-19 are based on previous experiences/ lessons gain from the SARS-CoV and MERS-CoV epidemics. Like other CoVs, SARS-CoV-2 has a positive-sense single-stranded RNA as genome and four structural proteins, namely the spike (S), envelope (E), membrane (M), and nucleocapsid (N) proteins. As for mechanism of the CoVs infection is concerned, similar to other CoVs, it uses their surface spike proteins and interact with its cognate receptor i.e angiotensin-converting enzyme 2 (ACE2) receptor present on the host cell surface. Unlike, other CoVs, SARS-CoV-2 spike protein has high affinity to the host cellular ACE2 receptor (Zhang, Penninger, Li, Zhong $\&$ Slutsky 2020). Most of the current research for the development of effective therapeutics 
against SARS-VCoV-2 are based on the screening of existing broad-spectrum anti-virals, small molecule libraries, FDA approved drug libraries and traditional alternative medicines (Maurya 2020; Maurya \& Sharma 2020; Wu et al. 2020; Zhou et al. 2020). Very recently, a team of the doctors from Bangladesh Medical College Hospital, has reported that Ivermectin given single dose with doxycycline, yielded the near-miraculous results in curing COVID-19 patients.

Ivermectin is a FDA-approved broad spectrum anti-parasitic drug (González Canga et al. 2008). It affects a plethora of invertebrate species, including parasitic arachnids, nematodes and insects. It has been earlier shown to exert anti-viral activity in vitro against Dengue fever virus (DENV), influenza virus, West Nile Virus and Venezuelan equine encephalitis virus (Lundberg et al. 2013; Tay et al. 2013; Götz et al. 2016). Very recently, Caly et al (2020) have shown the efficacy of the Ivermectin in Vero-hSLAM cells infection with SARS-CoV-2 and observed an effective loss of viral material within $48 \mathrm{~h}$ (Caly, Druce, Catton, Jans \& Wagstaff 2020). Based on their finding, Caly et al (2020) hypothesised that it might we working by inhibiting Importin $\alpha / \beta 1$ (IMP $\alpha / \beta 1)$-mediated nuclear import of viral proteins, as shown for other RNA viruses (Caly, Wagstaff \& Jans 2012; Lundberg et al. 2013; Tay et al. 2013; Caly et al. 2020; Yang et al. 2020). As for safety is concerned, Ivermectin has an established safety profile for human use (González Canga et al. 2008; Buonfrate et al. 2019). The second drug, doxycycline is a synthetic antibiotic derived from tetracycline and have broad spectrum antibiotic property. Doxycycline other than antibiotics, are known to inhibit M2-type polarization of macrophages (He \& Marneros 2014). Doxycycline is a FDA approved antibiotic since 1967 and is extensively referred by most clinicians. As for its safety is concerned, doxycycline has a long history of safety.

In the current study, we have explored the possible mechanisms to explain the above experimental and clinical findings using molecular docking and molecular dynamics simulation studies. For this, Ivermectin and doxycycline were docked with different viral proteins (such as spike, Mpro, PLpro, RNA dependent RNA polymerase, Nucleocapsid, NSP3, NSP10, NSP15 and NSP16) and host cell receptor (ACE2). Our molecular docking study predicted that Ivermectin and doxycycline both have significant binding affinity for SARS-CoV2 spike and ACE2 interacting region, indicating poor interaction for viral entry inside the cells. The binding affinity of the Ivermectin is much better than the doxycycline. At the same time, Ivermectin has significantly high binding affinity with the viral RNA dependent RNA polymerase (RdRp, NSP12), indicating poor viral genome replication for viral multiplication. Ivermectin significantly inhibited ADP Ribose Phosphatase (NSP3), Endoribonuclease (NSP15) and methyltransferase (NSP10-NSP16 complex) of SARS-CoV-2 which were involved in the virus escape from the host innate immune system. Ivermectin and doxycycline both bind with SARS-CoV-2 main protease (Mpro) adjacent to its active site. Our study provides the possible mechanism of the action of this combination and a scientific justification for their application in prevention and management of COVID-19 crisis.

\section{Materials and Methods}

Chemical structure of molecules and target proteins: Chemical structures information for Ivermectin and doxycycline were downloaded from PubChem (https://pubchem.ncbi.nlm.nih.gov). In order to study the mode of interaction of Ivermectin 
and doxycycline with various SARS-CoV-2 proteins and receptors found on host cells, following PDB ID's 6lu7 (SARS-CoV-2 Mpro), 6m0j (SARS-CoV-2 spike), 6m71 (SARS-CoV-2 RdRp, NSP12), 6w02 (NSP3), 6w9c (PLpro), 6m3m (Nucleocapsid), 6w4b (NSP9), 6wkq (NSP10), 6vww (NSP15), 6wkq (NSP16) and 6m0j (ACE2) were used. All the protein structures were retrieved from protein data bank (https://www.rcsb.org/) and cleaned using USCF Chimera software (Pettersen et al. 2004).

Molecular docking: PyRx virtual screening tool was used for preparation of the ligands and receptor to perform the molecular docking. Molecular docking was performing using Vina wizard of PyRx virtual screening tool (Dallakyan \& Olson 2015). For preparation of protein input files, all the water molecules, ligands and ions were removed from *.pdb files and polar hydrogens were added before saving *.pdbqt format. Both the molecules were energy minimized and saved in *.pdbqt format after adding polar hydrogens for further docking process. Following AutoDock Vina docking parameters such as (center_x $=-26.2834$, center_y $=12.5988$, center_z $=58.9648$, size_ $x=66.124$, size_ $y=22.9423$, size_z $=51.456)$, (center_ $x$ $=-32.483$, center_y $=26.077$, center_z $=7.923$, size_ $x=52.974$, size_y $=46.699$, size_z $=$ 30.699) and (center_x $=-25.0997$, center_y $=19.903$, center_z $=3.047$, size_ $x=78.872$, size_y $=67.603$, size_z $=35.866$ ) were used for SARS-CoV-2 Mpro (PDB ID: 6LU7), SARS-CoV-2 spike (PDB ID: 6m0j) and human ACE2 (PDB ID: 6m0j) respectively. For docking RdRp (PDB ID: 6m71) NSP7 and NSP8 was removed from the NSP12 and grid box for docking parameter was set at the NSP12-NSP7 and NSP12-NSP8 interface. Docking parameters used for NSP12 were center_ $x=112.289$, center_ $y=131.245$, center_z $=141.597$, size_ $x=83.597$, size_ $y=69.959$, size_z $=45.240$. A blind docking was performed for the remaining target receptor proteins.

Interactions of the ligand with critical residues: 2D interaction of docked complex were analysed for the visualization of the best binding poses. As the importance of hydrogen bonding is well documented in drug discovery and design, further hydrogen bond and other non-polar interactions of best binding poses were examined using Discovery studio software (Dassault Systèmes BIOVIA, San Diego, 2016).

Molecular dynamics simulation: The protein-ligand complex for spike, Mpro, NSP3, NSP16 and human ACE2 receptor with Ivermectin were obtained after the molecular docking study. The simulation systems for protein-ligand complex without or with the Ivermectin were performed using the GROMACS software (Abraham et al. 2015). Protein topology was prepared using pdb2gmx module of GROMACS. Whereas ligand topology was derived from Prodrg server (http://prodrg1.dyndns.org/)(van Aalten et al. 1996). MD simulation was performed with Gromos53a6.ff force-field using the GROMACS. Proteins were solvated using SPC (simple point charge) water model and counter ions were added to neutralize the system. After that, system was energy minimized and further water and ions were allowed to equilibrate around the protein in a two-step equilibration process. The first part of equilibration was at a constant number of particles, volume, and temperature (NVT). The second part of equilibration was at a constant number of particles, pressure, and temperature (NPT). NVT and NPT equilibration simulations were performed for 100 ps. Following 
equilibration, the production simulation duration was performed for 5000 picoseconds (ps). The trajectory data were saved at different time points to analyse the change in the dynamics of protein-ligand complex. After completion of MD simulation, results for flexibility for protein-ligand complexes were analysed using root mean square deviation (RMSD) and root mean square fluctuation (RMSF) using GROMACS.

\section{Results and Discussion}

Different therapeutics have different targets for the prevention of the CoVs severity (Prajapat et al. 2020). Some may block virus spike interaction with host cell receptors, or some may interfere with viral genome replication and some other may obstruct the process of virus assembly (Prajapat et al. 2020). In the current study, drugs Ivermectin and doxycycline were evaluated for their binding affinities against SARS-CoV-2 spike, Mpro, PLpro, RdRp, nucleocapsid, NSP3, NSP9, NSP10, NSP15, NSP16 and human ACE2 receptor by molecular docking. Further binding stability of spike, Mpro, NSP3, NSP16 and ACE2 protein complexed with Ivermectin were evaluated by molecular dynamics simulation.

Molecular docking: Our molecular docking study revealed that both Ivermectin and doxycycline have significant binding affinity with various SARS-CoV-2 proteins i.e Mpro, spike, PLpro, RdRp, nucleocapsid, NSP3, NSP9, NSP10, NSP15, NSP16 and host ACE2 receptor (Table 1). Ivermectin showed better binding affinity with these target proteins compared to doxycycline. During SARS-CoV-2 infection, interaction of spike-RBD protein with the host cell receptor facilitate virus invasion and determine viral tissue or host tropism (Li 2016). Initial interactions of spike with host receptor (ACE2), and subsequent fusion of the host and viral membrane allows the viral genome to enter inside the host cells (Li 2016). It is now well established that, amino acid residues SER19, GLN24, THR27, PHE28, ASP30, LYS31, HIS34, GLU35, GLU37, ASP38, TYR41, GLN42, LEU45, LEU79, MET82, TYR83, ASN330, LYS353, GLY354, ASP355 and ARG357 of the human ACE2 interact with amino acid residues LYS417, GLY446, TYR449, TYR453, LEU455, PHE456, TYR473, ALA475, GLY476, GLU484, PHE486, ASN487, TYR489, PHE490, GLN493, GLY496, GLN498, THR500, ASN501, GLY502 and TYR505 of SARS-CoV-2 spike-RBD protein (Wang et al. 2020). Our results show that, Ivermectin binds at the junction of spike-RBD and ACE2 interaction site indicating that it might have potential to inhibit the entry of the virus to the host cell (Fig 1a). Different amino acid residues of spike protein interacting with Ivermectin are ARG403, ASP405, ARG408, GLN409, GLY416, LYS417, TYR449, TYR453, LEU455, PHE456, SER494, TYR495, GLY496, GLN498 and TYR505 whereas amino acid interacting with ACE2 includes LYS26, LEU29, ASP30, ASN33 HIS34, GLU37, THR92, VAL93, GLN96, ALA386, ALS387, GLN388, PRO389, LEU392, ARG393, ARG559 and SER563. From the Figure $1 \mathrm{~A}$, it is clear that Ivermectin independently bind the interacting region of viral spike and host ACE2 receptor indicating that it might hamper the interaction of spike and ACE2 receptor and inhibit the virus entry inside the cells. Figure $1 B$, shows the $2 D$ interaction of Ivermectin and doxycycline with different protein residues of spike and ACE2. The 
predicted binding energies of Ivermectin and doxycycline are -7.2 and $-6.6 \mathrm{kcal} / \mathrm{mol}$ for spike protein and -7.5 and $-7.6 \mathrm{kcal} / \mathrm{mol}$ for ACE2 receptor respectively.

Table 1: Predicted binding energy and inhibition constant of Ivermectin and Doxycycline for various SARS-COV-2 target proteins and human ACE2 receptor

\begin{tabular}{|c|c|c|c|}
\hline Drugs & Targets, PDB ID's & $\begin{array}{l}\text { Predicted binding } \\
\text { energy (kcal/mol) }\end{array}$ & $\begin{array}{l}\text { Predicted Inhibition } \\
\text { constant, } \mathrm{Ki}(\mu \mathrm{M})\end{array}$ \\
\hline \multirow[t]{11}{*}{ Ivermectin } & Mpro, 6lu7 & -8.4 & 0.69 \\
\hline & PLpro, 6w9c & -8.5 & 0.58 \\
\hline & Spike, $6 \mathrm{m0j}$ & -7.2 & 5.21 \\
\hline & Nucleocapsid, $6 \mathrm{~m} 3 \mathrm{~m}$ & -8.2 & 0.96 \\
\hline & NSP3, 6w02 & -8.0 & 1.35 \\
\hline & NSP9, 6w4b & -7.9 & 1.60 \\
\hline & NSP10, 6wkq & -8.9 & 0.69 \\
\hline & NSP12, 6m71 & -9.1 & 0.21 \\
\hline & NSP15, 6vww & -8.4 & 0.69 \\
\hline & NSP16, 6wkq & -8.3 & 0.81 \\
\hline & ACE2, 6m9j & -7.5 & 3.14 \\
\hline \multirow[t]{11}{*}{ Doxycycline } & Mpro, 6lu7 & -7.1 & 6.16 \\
\hline & PLpro, 6w9c & -7.1 & 6.16 \\
\hline & Spike, $6 \mathrm{m0j}$ & -6.6 & 14.35 \\
\hline & Nucleocapsid, 6m3m & -6.3 & 23.82 \\
\hline & NSP3, 6w02 & -7.1 & 6.16 \\
\hline & NSP9, 6w4b & -6.1 & 33.40 \\
\hline & NSP10, 6wkq & -6.9 & 8.64 \\
\hline & NSP12, 6m71 & -7.9 & 1.60 \\
\hline & NSP15, 6vww & -7.5 & 3.14 \\
\hline & NSP16, 6wkq & -7.7 & 2.24 \\
\hline & ACE2, 6m9j & -7.6 & 2.65 \\
\hline
\end{tabular}

The RdRp is the fundamental component of SARS-CoV-2 replication/transcription machinery. Figure 2, shows the predicted binding site and 2D interaction of Ivermectin and doxycycline for SARS-CoV-2 RdRp. The anticipated binding energies of Ivermectin and doxycycline for RdRp are -9.1 and $-7.9 \mathrm{kcal} / \mathrm{mol}$ respectively. SARS-CoV-2 Mpro is considered as a promising druggable target because it plays vital role in viral gene expression and replication and are essential for maturation cleavage events of viral precursor polyprotein. The critical maturation cleavage events within the viral precursor polyprotein are mediated by the Mpro. Figure 3, shows the predicted binding site and 2D interaction of Ivermectin and doxycycline with SARS-CoV-2 Mpro. The predicted binding energies of Ivermectin and doxycycline for Mpro are -8.4 and $-7.1 \mathrm{kcal} / \mathrm{mol}$ respectively. Our study shows that although both Ivermectin and doxycycline have strong binding affinity with the Mpro, but they bind off-site to the active site of the Mpro indicating they may not directly inhibit Mpro rather will decrease their activity by interfering with its flexibility (Figure 3 ). 
Figure 1: Interaction of Ivermectin and doxycycline with SARS-COV-2 spike and human ACE2 receptor A. Interaction of Ivermectin with SARS-CoV-2 spike and human ACE2 receptor.

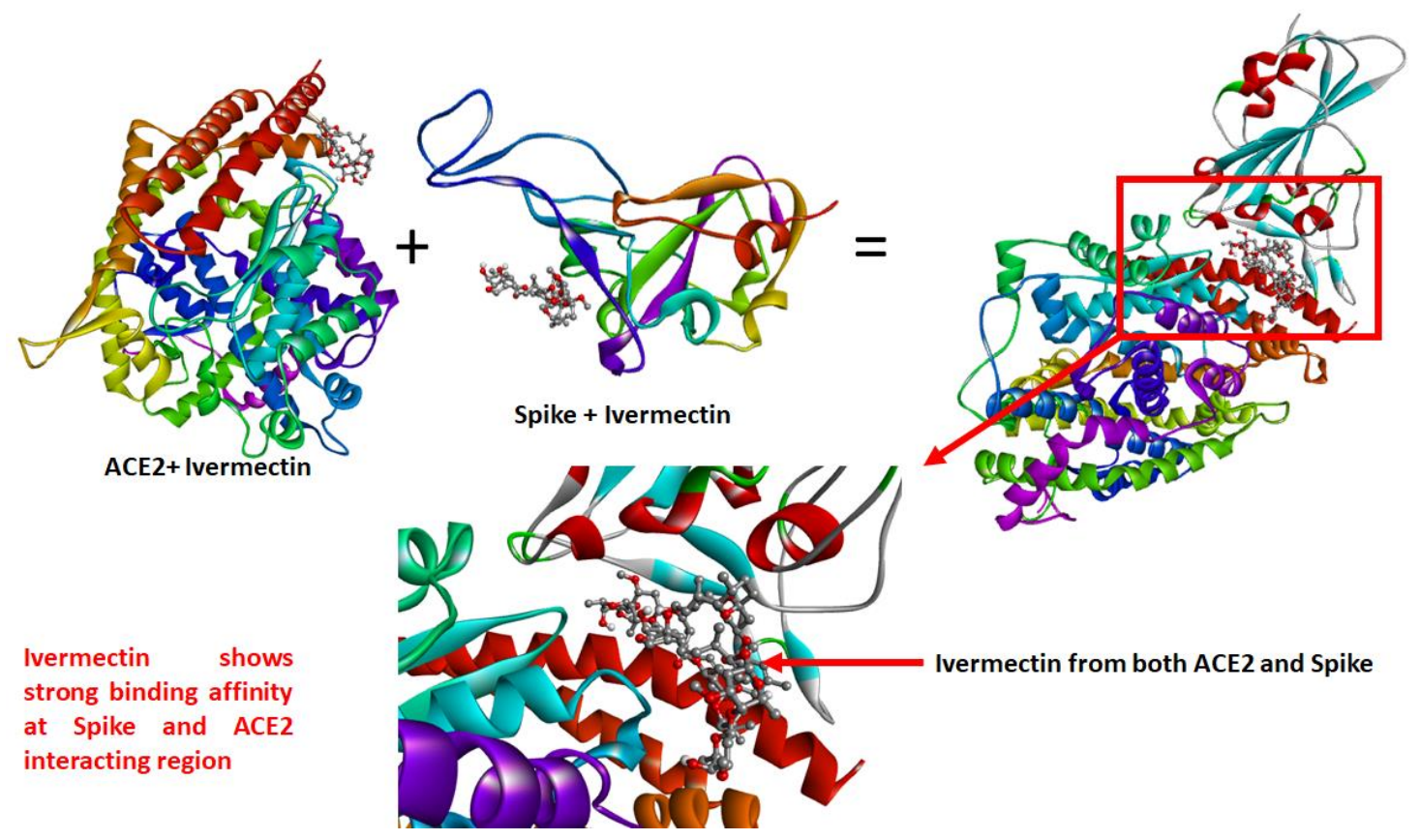

B. 2D interaction of Ivermectin and doxycycline with SARS-COV-2 spike and human ACE2 receptor. 

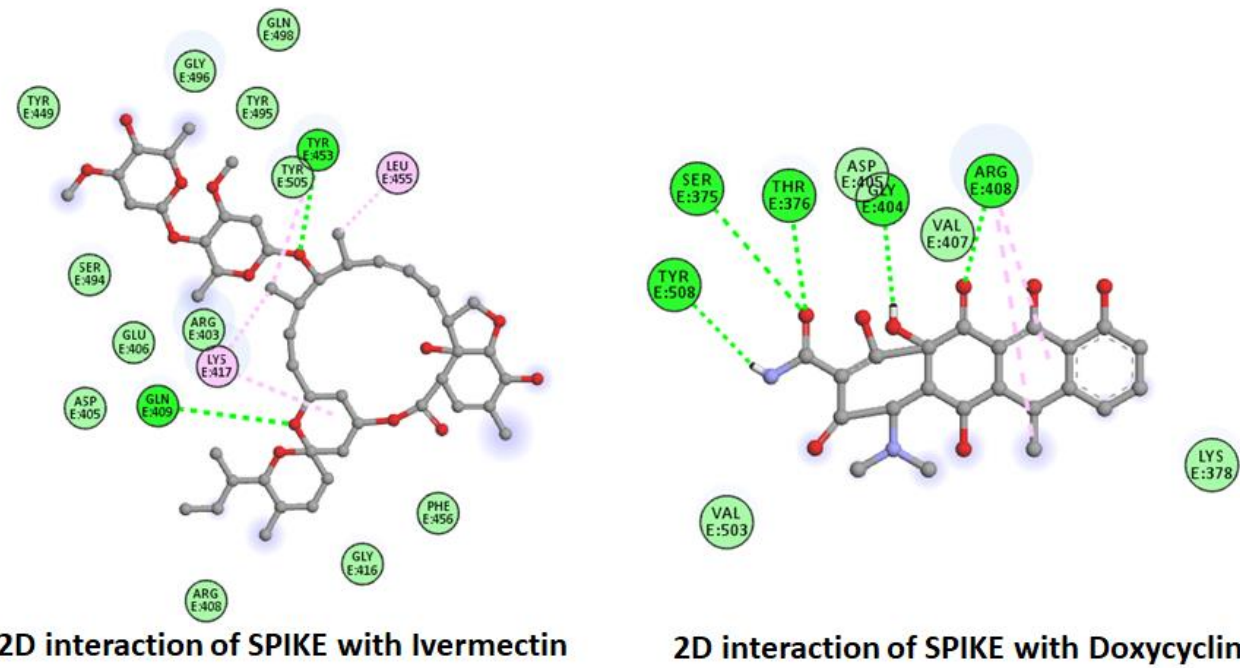

2D interaction of SPIKE with Ivermectin

2D interaction of SPIKE with Doxycycline
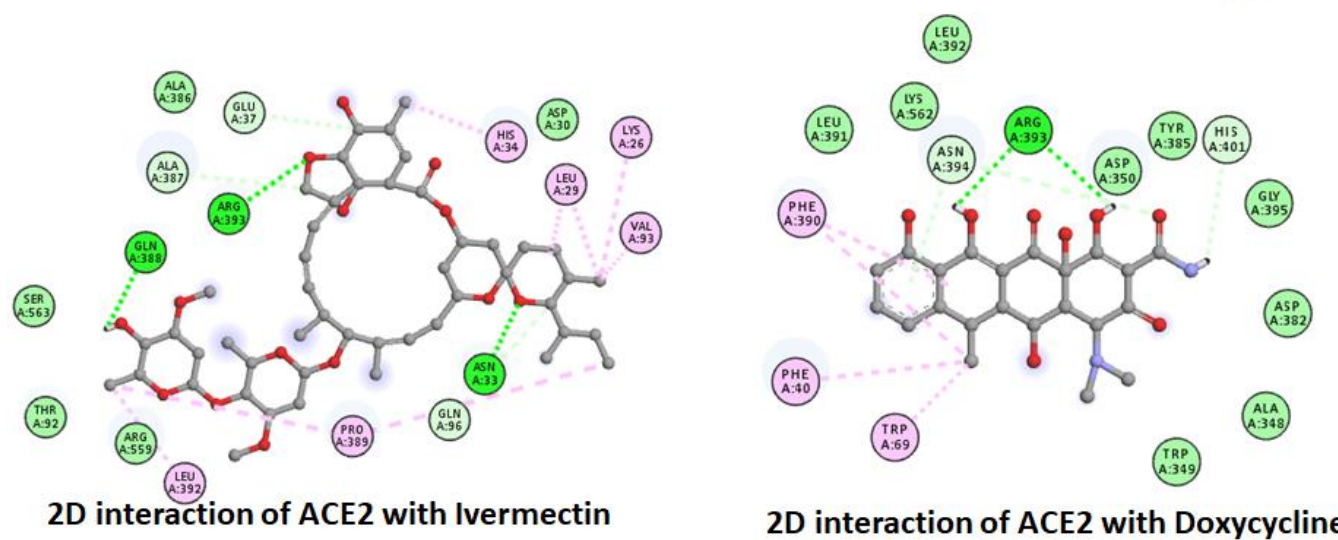

Figure 2: Interaction of Ivermectin and doxycycline with SARS-CoV-2 RdRp.

A. Binding site of Ivermectin and doxycycline with SARS-CoV-2 RdRp.

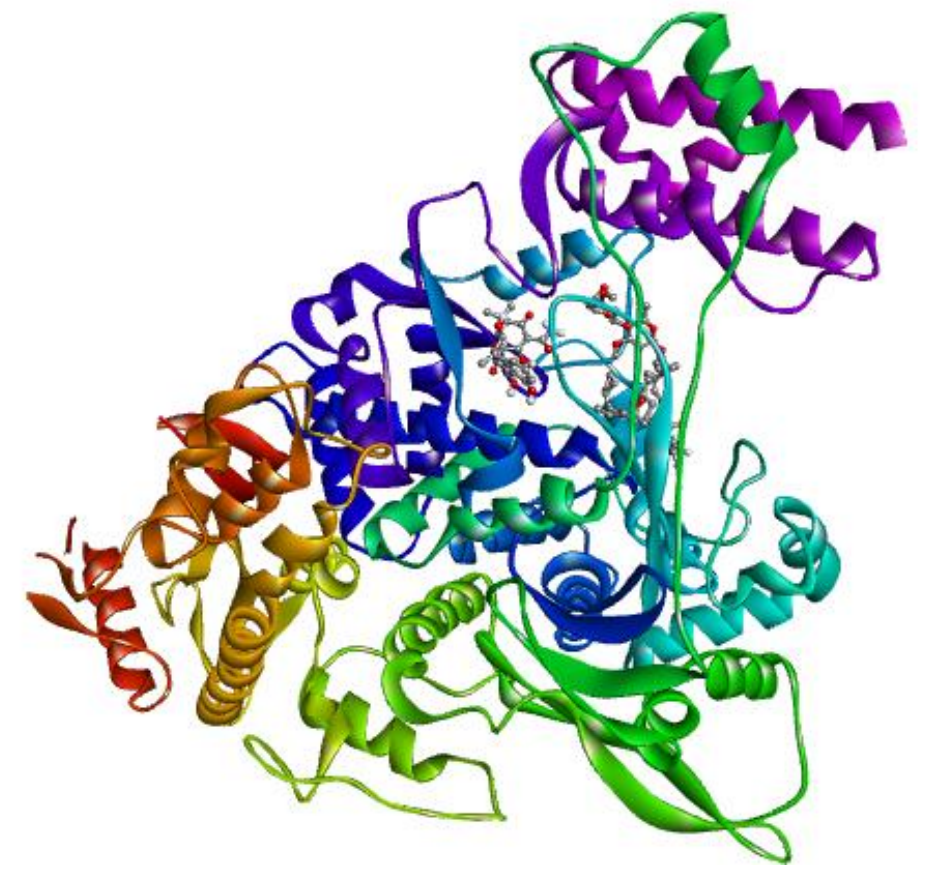


B. 2D interaction of Ivermectin and doxycycline with SARS-CoV-2 RdRp.
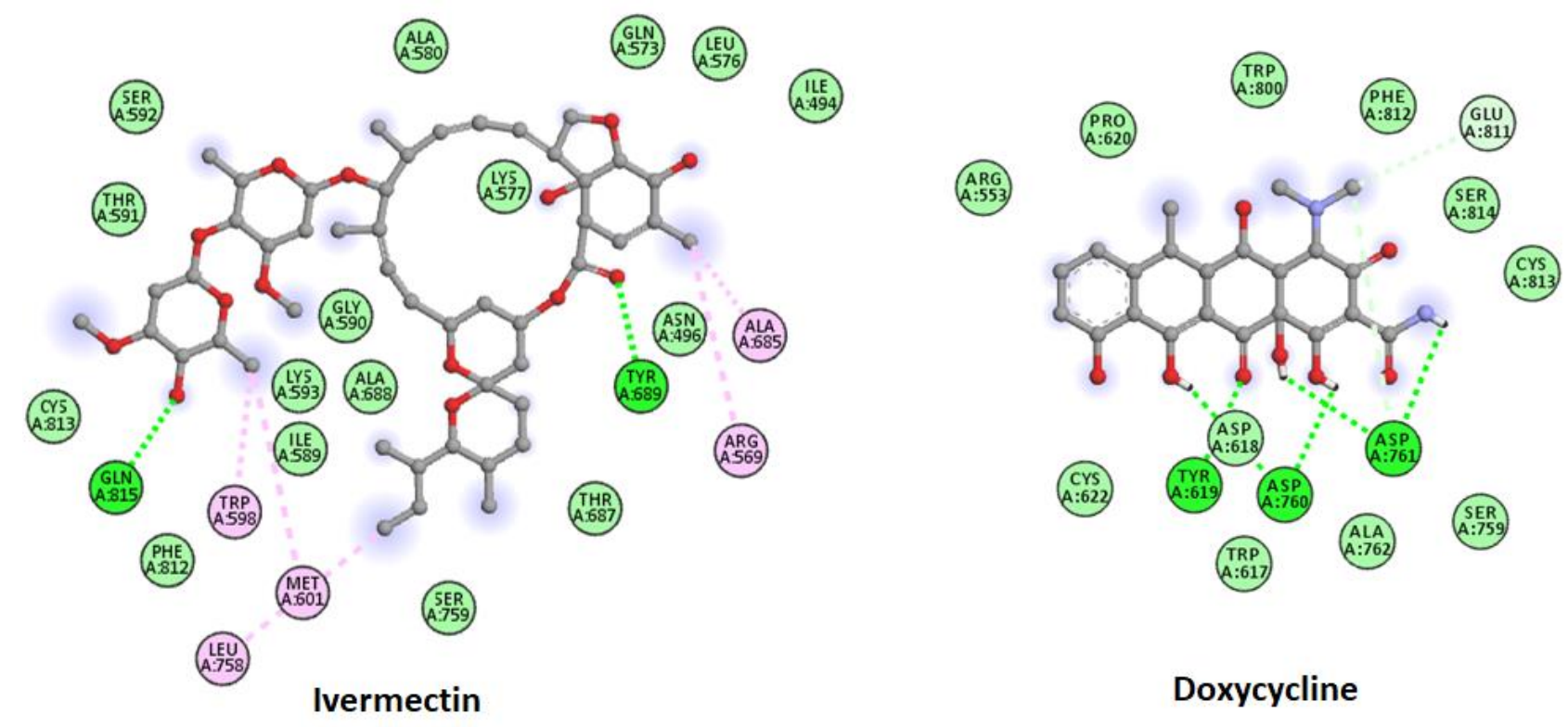

Doxycycline

Figure 3. Interaction of Ivermectin and doxycycline with SARS-COV-2 Mpro.

A. Binding site of Ivermectin and doxycycline with SARS-CoV-2 Mpro.

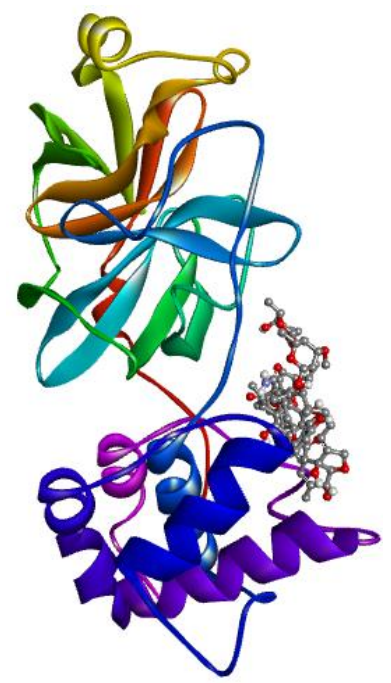

B. 2D interaction of Ivermectin and doxycycline with SARS-CoV-2 Mpro. 


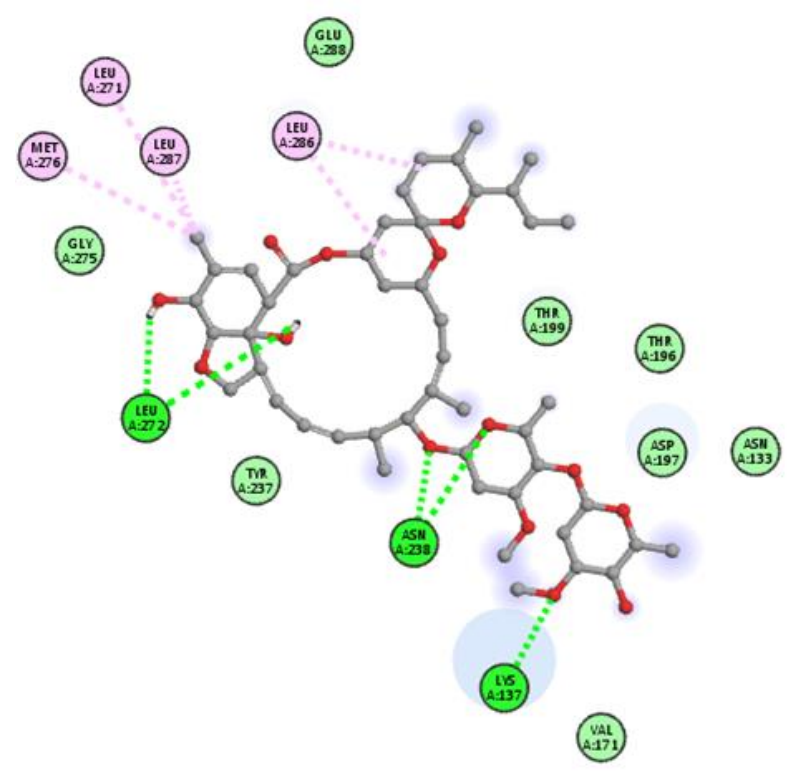

Ivermectin

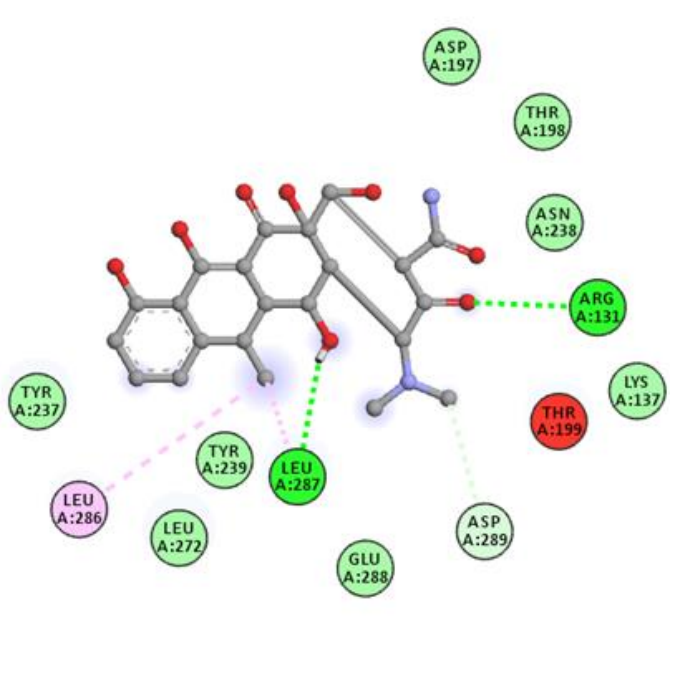

Doxycycline

The NSP3 is the largest multi-domain protein encoded by the CoVs and is a vital component of the replication/transcription complex (RTC). NSP3 function as an ADP-ribose-1'phosphatase. Besides its macrodomain, NSP3 also contains a papain-like protease (PLpro) required during the processing of the CoVs NSPs. Ubiquitination is a posttranslational modification that, among many other functions, is very important in the regulation of innate immune pathways (Mielech, Chen, Mesecar \& Baker 2014). PLpro is believed to antagonize the IFN response through its de-ubiquitinating enzyme activity, which allows the protease to remove ubiquitin from a substrate. Figure 4, shows the predicted binding site and 2D interaction of Ivermectin and doxycycline with SARS-CoV-2 NSP3 and PLpro. The predicted binding energies of Ivermectin and doxycycline -8.0 and $-7.1 \mathrm{kcal} / \mathrm{mol}$ for NSP3 whereas -8.5 and $-7.1 \mathrm{kcal} / \mathrm{mol}$ for PLpro. These observations show that, binding of Ivermectin with NSP3 and PLpro may block its enzymatic activity and thereby prevent SARS-CoV-2 from downregulating the anti-viral interferon response. This may improve viral load clearance by host innate immune system and reduce the extent of viral pathogenesis in COVID-19 patients without any previous memory of the antigen.

Viral RNAs are capped to safeguard their stability, efficient translation, and evading the innate immune system of the host cell. The RNA capping tactic are employed by several RNA viruses to avoid immune detection by toll-like receptor 7 (TLR7) and toll-like receptor 8 (TLR8) (Hyde \& Diamond 2015). The viral RNA capping machinery is anatomically and mechanistically different compared to eukaryotic mRNA capping system. The CoVs NSP16 protein is a RNA cap modifying enzyme and become active when complexed with its activating partner NSP10 (Decroly et al. 2011). NSP16 binds with NSP10 resulting a complex which exhibits RNA cap (nucleoside-2'-O)-methyltransferase activity. Figure 5, shows the predicted binding site and 2D interaction of Ivermectin and doxycycline with SARS-CoV-2 NSP16 and NSP10. Our study 
shows that Ivermectin binds to the S-Adenosylmethionine binding site of NSP16 with a binding affinity of $-8.3 \mathrm{kcal} / \mathrm{mol}$. It also binds with the NSP16 cognate activation partners NSP10 with a binding affinity of $-8.9 \mathrm{kcal} / \mathrm{mol}$. This indicates that the binding of Ivermectin to the S-Adenosylmethionine binding site will hamper the essential methyl-group transfer and inhibit the methyltransferase activity of NSP16. In the absence of methyl group attachment to the 5'cap of viral RNA, it will get easily detected by host innate immune system. Strong binding affinity of Ivermectin with NSP10 may also interfere in the formation of NSP16-NSP10 complex.

Some of the other important CoVs proteins such as nucleocapsid (N), NSP9 and NSP15 were also used for molecular docking study. The CoVs $\mathrm{N}$ protein is a structural protein that forms complexes with viral genome, interacts with the viral membrane protein during virion assembly and plays an important role in enhancing the efficiency of virus transcription and assembly (McBride, van Zyl \& Fielding 2014). N-terminal region of nucleocapsid protein consists of positively charged amino acids, which are responsible for its RNA binding. CoVs NSP9 is most likely involved in viral RNA synthesis. NSP9 binds with RNA and interacts with NSP8 to execute its essential functions (Sutton et al. 2004). Dimeric form of NSP9 is important

Figure 4. Interaction of Ivermectin and doxycycline with SARS-CoV-2 NSP3 and PLpro.

A. Binding site of Ivermectin and doxycycline with SARS-COV-2 NSP3 and their 2D interactions. 


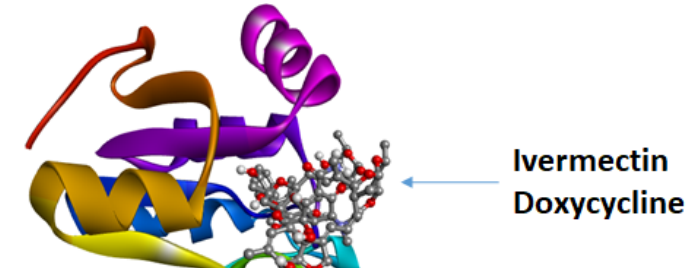

ALA

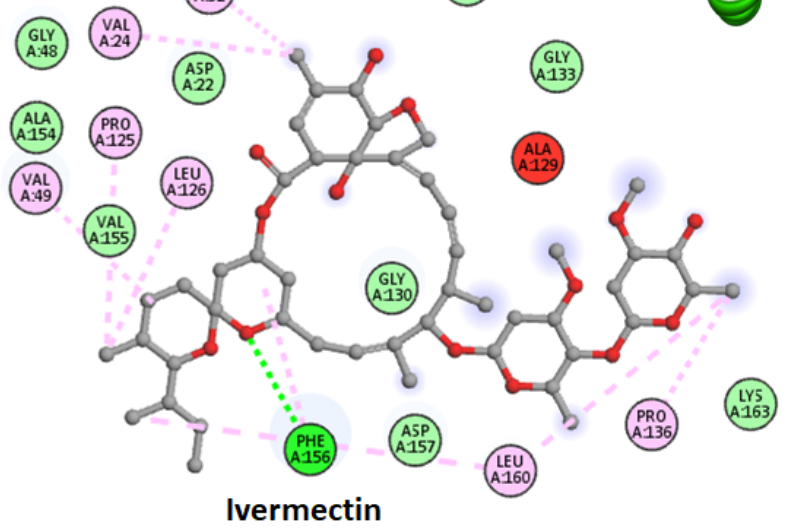

ILE

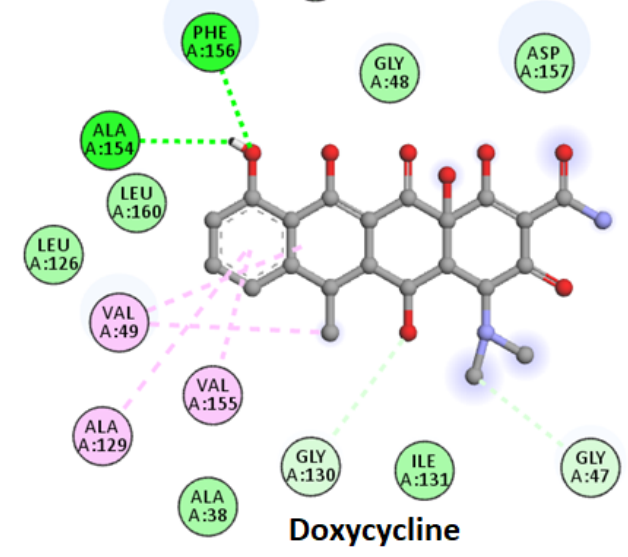

B. Binding site of Ivermectin and doxycycline with SARS-COV-2 PLpro and their 2D interactions.

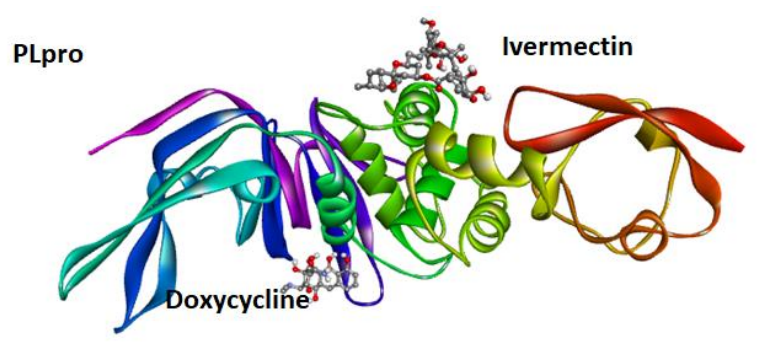

A5P

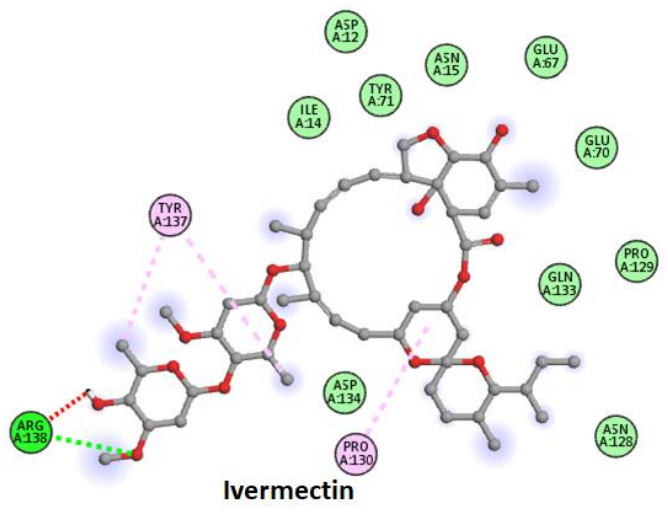

VAL

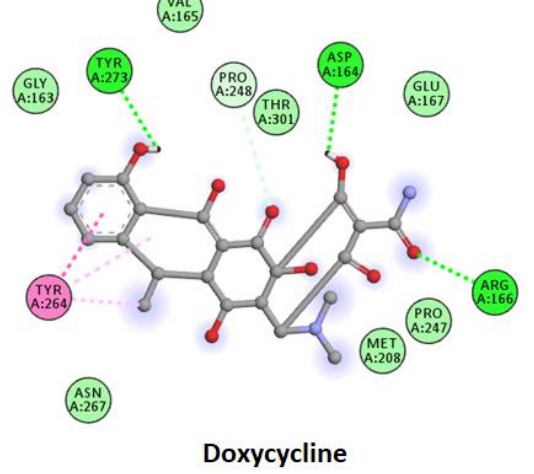

Figure 5. Interaction of Ivermectin and doxycycline with SARS-CoV-2 NSP16 and NSP10.

A. Binding site of Ivermectin and doxycycline with SARS-CoV-2 NSP16 and their 2D interactions. 


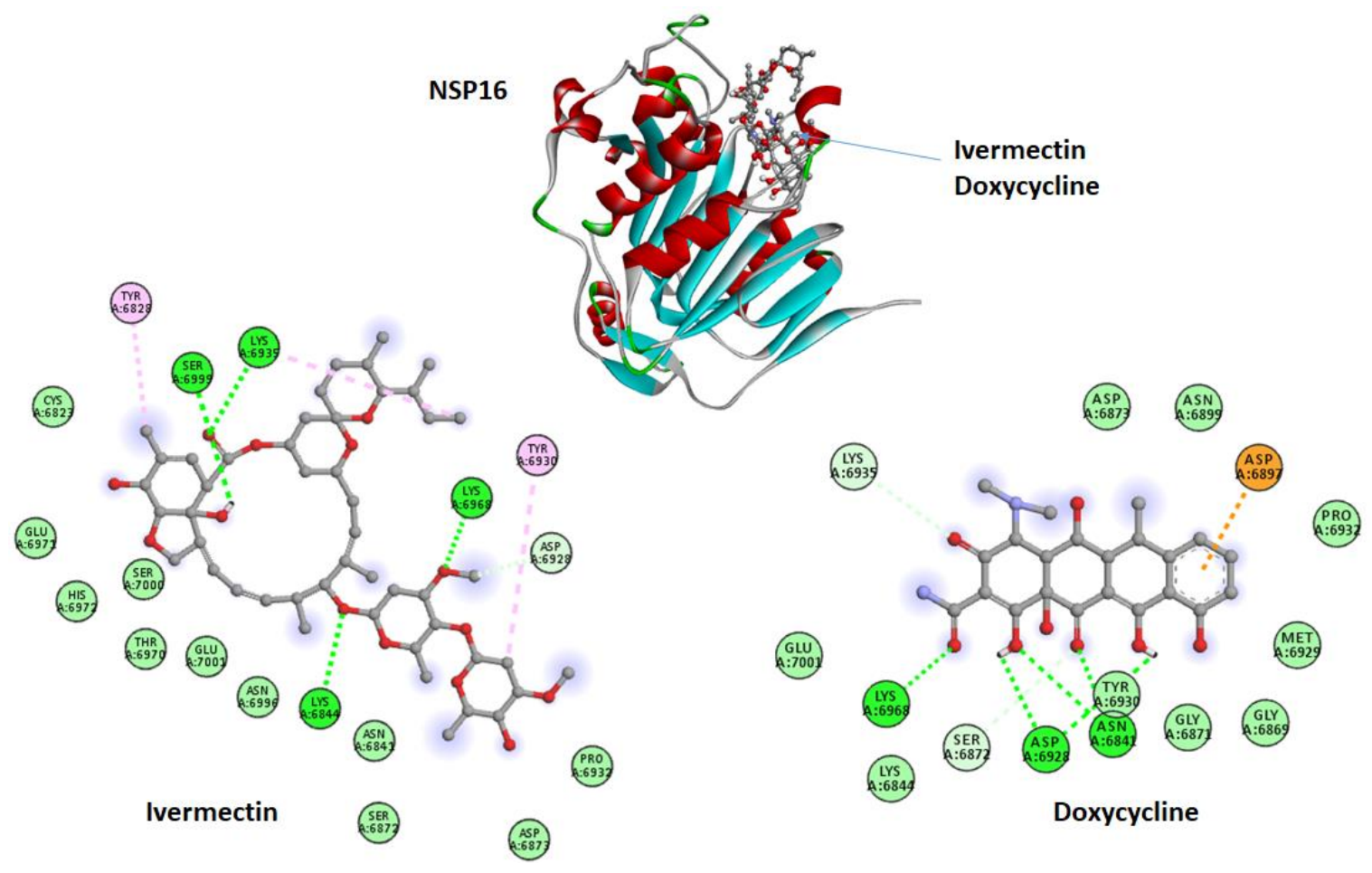

B. Binding site of Ivermectin and doxycycline with SARS-CoV-2 NSP16 and their 2D interactions.

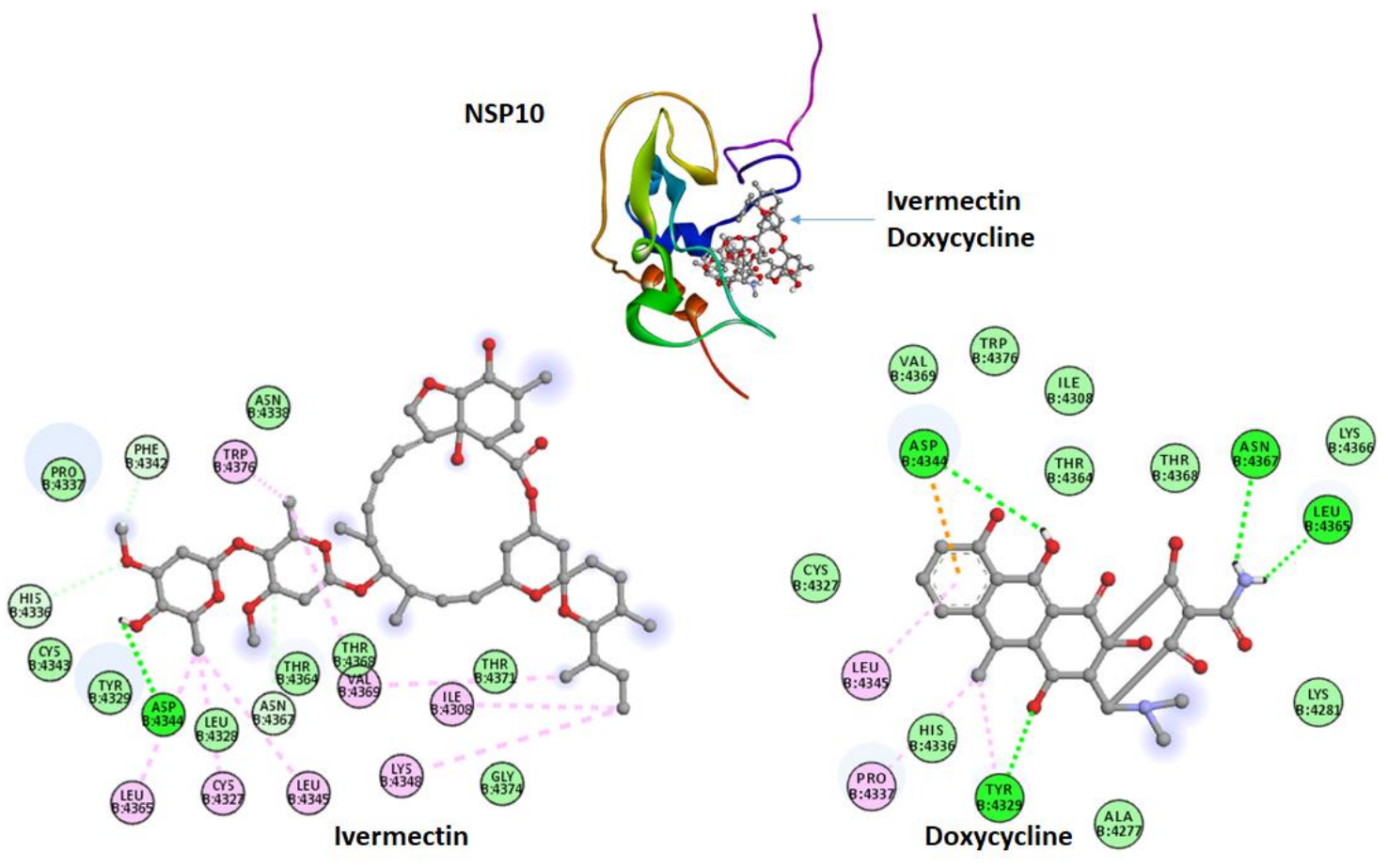

for viral infection. Any disturbance in the dimerization of NSP9 can be a way to overcome CoVs infection (Egloff et al. 2004; Hu et al. 2017). Whereas the function of NSP15 has a poorly defined but it was observed that a mutations in the active site of NSP15 abolish its endoribonuclease activity in vitro and reduces viral infectivity (Posthuma et al. 2006; Kang et 
al. 2007). At the same time, any mutations outside of the active site are reported to have a larger effect on virus viability, indicating that NSP15 has role in CoVs infection apart from its function as an endoribonuclease (Ivanov et al. 2004; Kang et al. 2007; Bhardwaj, Liu, Leibowitz \& Kao 2012). In our study, predicted binding energies of Ivermectin and doxycycline for $\mathrm{N}$ protein are -8.2 and $-6.3 \mathrm{kcal} / \mathrm{mol}$ respectively. The predicted binding energies of Ivermectin and doxycycline are -7.9 and $-6.1 \mathrm{kcal} / \mathrm{mol}$ for NSP9 whereas -8.4 and $-7.5 \mathrm{kcal} / \mathrm{mol}$ for NSP15. These finding show that Ivermectin have better binding affinity with N-protein, NSP9, and NSP15 compared to doxycycline. Altogether binding of Ivermectin with these proteins will hamper their individual function and decreases the viral genome replication, processing of virion packaging and ultimately viral load.

Molecular dynamics simulation: MD simulation is helpful in studying the stability of binding pose gained from docking, to rescore the inclusive binding energy as well as energy contribution by different residues, to study the conformational variations happening in protein due to ligand binding/unbinding etc. Thus, MD simulation was performed to examine the stability and dynamics of protein to confirm the docking results and get more insight into the stability of ligand-protein complex. MD simulations were carried out for SARS-CoV-2 spike, Mpro, NSP3, NSP16 and human ACE2 receptor and Ivermectin complex in the solvated states for 5000 ps. The results of MD simulations have been examined on the basis of root mean square deviation (RMSD) and root mean square fluctuation (RMSF). Based on the obtained MD trajectories, RMSD values of the protein backbone in complexes with the Ivermectin was computed. In all the cases, the RMSD values steadily increased from 0 to 200 ps, and reached equilibrium after that throughout the simulation period. RMSD was plotted with respect to time and showed stable behaviour throughout the simulation (Figure 6A). To explore the local protein flexibility, the time average of RMSF values of spike, Mpro, NSP3, NSP16 and ACE2 protein in presence Ivermectin over simulation period were calculated. RMSF with respect to amino acid was plotted and found no major fluctuation except few places where moderate fluctuation was observed (Figure 6B).

Most of the CoVs are known to bind with host metalloproteases (MMPs), predominantly for viral survival. Tetracyclines are known to chelate Zinc from MMPs (Aggarwal et al. 2010). Doxycycline being a tetracycline derivative, it will be beneficial in inhibition of SARS-CoV-2 infection because it chelates Zinc (Phillips, Gallagher \& Weiss 2017). Tetracyclines also possess anti-viral activities and known to modulate innate immunity. Anti-viral activity of doxycycline had already been reported against retroviruses and dengue virus (Sturtz 1998; Yang, Chen, Tu, Yen \& Yang 2007). They can decrease the expression of NF-kB and the release of inflammatory cytokines such as TNF- $\alpha, \mathrm{IL}-1 \beta$ and IL-6, independent of their antibiotic mechanism (Henehan, Montuno \& De Benedetto 2017). Thus, they could participate in limiting the 'cytokine storm' induced in COVID-19 patients. The lipophilic nature of tetracyclines and their strong pulmonary penetration could allow them to inhibit viral replication at cellular level. 
Figure 6. Molecular dynamics simulation of Ivermectin complexed with Spike, ACE2, Mpro NSP16 and NSP3 proteins.

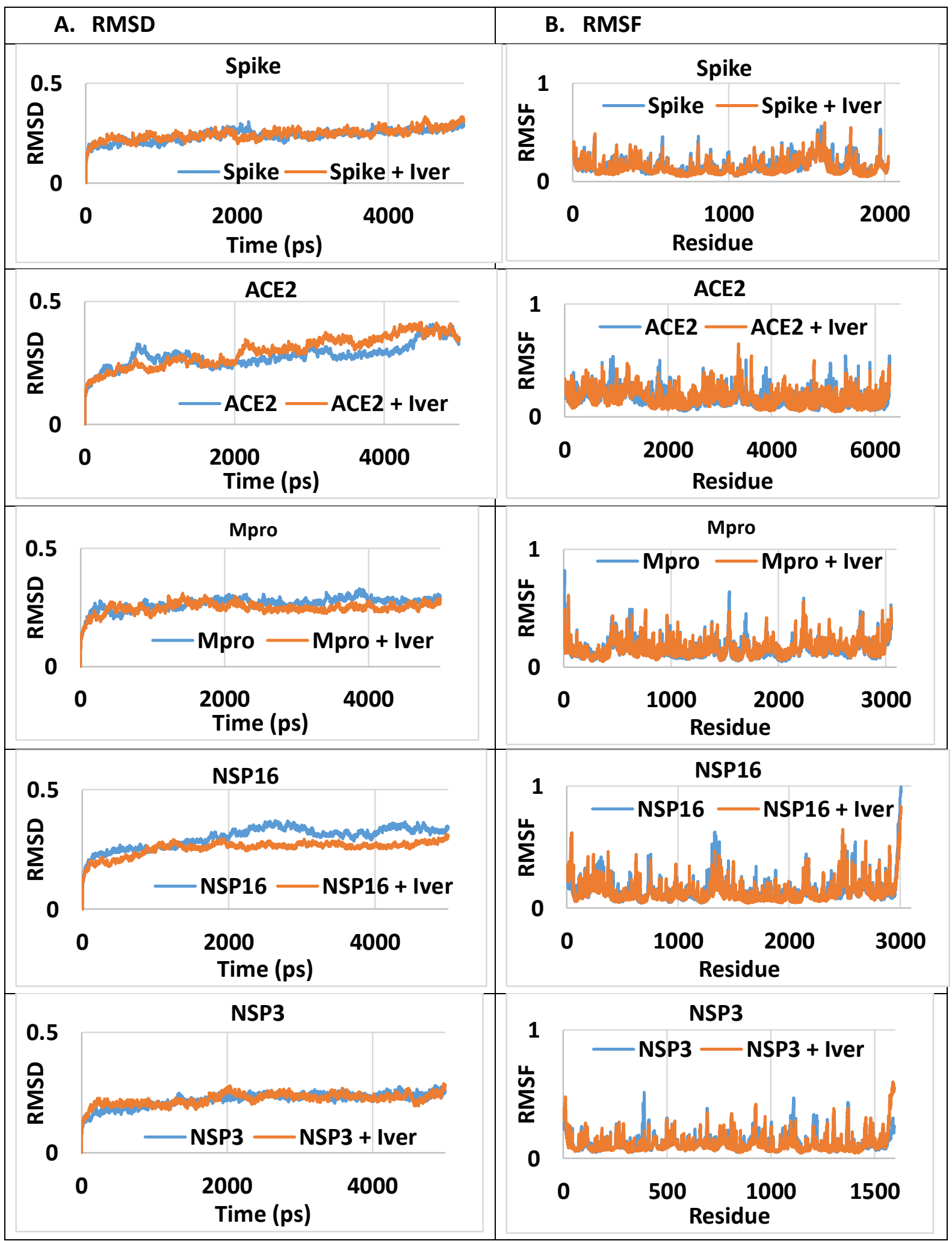

Among tetracyclines, doxycycline has unique advantages such as long history of safety, the short duration of treatment and low cost. At the same time Ivermectin possess anti-viral activity for a range of the viruses. Very recently, it was observed that Ivermectin significantly inhibited SARS-CoV-2 under in vitro conditions (Caly et al. 2020). 
In summary, the miraculous effect of combination of Ivermectin and doxycycline in COVID-19 patients is possibly by inhibition of spike-ACE2 interaction and inhibiting RNA dependent RNA polymerase, ADP Ribose Phosphatase, Endoribonuclease and NSP10-NSP16 complex mediated methyltransferase activities, anti-viral activity and chelation of the zinc \& immunomodulatory property. Thus, the usage of Ivermectin and doxycycline combination will be an ideal choice in prevention and management of COVID-19.

\section{Acknowledgement}

The author would like to thank Dr S. Santosh Kumar, Radiation Biology \& Health Sciences Division, Bhabha Atomic Research Centre, Mumbai for his valuable constructive suggestions and critical review of the article.

\section{Declaration of Conflicting Interests}

The author declared no potential conflicts of interest with respect to the research, authorship, and/or publication of this article.

\section{References}

Abraham, M. J., Murtola, T., Schulz, R., Páll, S., Smith, J. C., Hess, B. \& Lindahl, E. (2015). GROMACS: High performance molecular simulations through multi-level parallelism from laptops to supercomputers. SoftwareX 1-2: 19-25. https://doi.org/10.1016/j.softx.2015.06.001

Aggarwal, H. K., Jain, D., Talapatra, P., Yadav, R. K., Gupta, T. \& Kathuria, K. L. (2010). Evaluation of role of doxycycline (a matrix metalloproteinase inhibitor) on renal functions in patients of diabetic nephropathy. Ren Fail 32: 941-946. DOI: 10.3109/0886022X.2010.502606

Bhardwaj, K., Liu, P., Leibowitz, J. L. \& Kao, C. C. (2012). The coronavirus endoribonuclease Nsp15 interacts with retinoblastoma tumor suppressor protein. Journal of virology 86: 4294-4304. DOI: 10.1128/JVI.07012-11

Buonfrate, D., Salas-Coronas, J., Muñoz, J., Maruri, B. T., Rodari, P., Castelli, F., Zammarchi, L., Bianchi, L., Gobbi, F., Cabezas-Fernández, T., Requena-Mendez, A., Godbole, G., Silva, R., Romero, M., Chiodini, P. L. \& Bisoffi, Z. (2019). Multiple-dose versus single-dose ivermectin for Strongyloides stercoralis infection (Strong Treat 1 to 4): a multicentre, open-label, phase 3, randomised controlled superiority trial. The Lancet Infectious Diseases 19: 1181-1190. https://doi.org/10.1016/S1473-3099(19)30289-0

Caly, L., Druce, J. D., Catton, M. G., Jans, D. A. \& Wagstaff, K. M. (2020). The FDA-approved drug ivermectin inhibits the replication of SARS-CoV-2 in vitro. Antiviral Research 178: 104787. https://doi.org/10.1016/j.antiviral.2020.104787 
Caly, L., Wagstaff, K. M. \& Jans, D. A. (2012). Nuclear trafficking of proteins from RNA viruses: Potential target for antivirals? Antiviral Research 95: 202-206. https://doi.org/10.1016/j.antiviral.2012.06.008

Dallakyan, S. \& Olson, A. J. (2015). Small-molecule library screening by docking with PyRx. Methods Mol Biol 1263: 243-250. DOI: 10.1007/978-1-4939-2269-7_19

Egloff, M.-P., Ferron, F., Campanacci, V., Longhi, S., Rancurel, C., Dutartre, H., Snijder, E. J., Gorbalenya, A. E., Cambillau, C. \& Canard, B. (2004). The severe acute respiratory syndrome-coronavirus replicative protein nsp9 is a single-stranded RNA-binding subunit unique in the RNA virus world. Proceedings of the National Academy of Sciences of the United States of America 101: 3792-3796. https://doi.org/10.1073/pnas.0307877101

González Canga, A., Sahagún Prieto, A. M., Diez Liébana, M. J., Fernández Martínez, N., Sierra Vega, M. \& García Vieitez, J. J. (2008). The Pharmacokinetics and Interactions of Ivermectin in Humans-A Mini-review. The AAPS Journal 10: 42-46. DOI: 10.1208/s12248-007-9000-9

Götz, V., Magar, L., Dornfeld, D., Giese, S., Pohlmann, A., Höper, D., Kong, B.-W., Jans, D. A., Beer, M., Haller, O. \& Schwemmle, M. (2016). Influenza A viruses escape from MxA restriction at the expense of efficient nuclear VRNP import. Scientific reports 6: 23138-23138. DOI: 10.1038/srep23138

He, L. \& Marneros, A. G. (2014). Doxycycline inhibits polarization of macrophages to the proangiogenic M2-type and subsequent neovascularization. The Journal of biological chemistry 289: 80198028. DOI: $10.1074 / j b c . M 113.535765$

Henehan, M., Montuno, M. \& De Benedetto, A. (2017). Doxycycline as an anti-inflammatory agent: updates in dermatology. J Eur Acad Dermatol Venereol 31: 1800-1808. https://doi.org/10.1111/jdv.14345

Hu, T., Chen, C., Li, H., Dou, Y., Zhou, M., Lu, D., Zong, Q., Li, Y., Yang, C., Zhong, Z., Singh, N., Hu, H., Zhang, R., Yang, H. \& Su, D. (2017). Structural basis for dimerization and RNA binding of avian infectious bronchitis virus nsp9. Protein Sci 26: 1037-1048. DOI: 10.1002/pro.3150

Ivanov, K. A., Hertzig, T., Rozanov, M., Bayer, S., Thiel, V., Gorbalenya, A. E. \& Ziebuhr, J. (2004). Major genetic marker of nidoviruses encodes a replicative endoribonuclease. Proc Natl Acad Sci U S A 101: 12694-12699. DOI: 10.1073/pnas.0403127101

Kang, H., Bhardwaj, K., Li, Y., Palaninathan, S., Sacchettini, J., Guarino, L., Leibowitz, J. L. \& Kao, C. C. (2007). Biochemical and genetic analyses of murine hepatitis virus Nsp15 endoribonuclease. J Virol 81: 13587-13597. DOI: 10.1128/JVI.00547-07

Li, F. (2016). Structure, Function, and Evolution of Coronavirus Spike Proteins. Annu Rev Virol 3: 237261. DOI: 10.1146/annurev-virology-110615-042301

Lundberg, L., Pinkham, C., Baer, A., Amaya, M., Narayanan, A., Wagstaff, K. M., Jans, D. A. \& Kehn-Hall, K. (2013). Nuclear import and export inhibitors alter capsid protein distribution in mammalian cells and reduce Venezuelan Equine Encephalitis Virus replication. Antiviral Res 100: 662-672. DOI: 10.1016/j.antiviral.2013.10.004 
Maurya, D. K. (2020). Evaluation of Yashtimadhu (Glycyrrhiza glabra) active Phytochemicals Against Novel Coronavirus (SARS-CoV-2). Research Square (Preprint): doi.org/10.21203/rs.21203.rs26480/v21201+.

Maurya, D. K. \& Sharma, D. (2020). Evaluation of Traditional Ayurvedic Preparation for Prevention and Management of the Novel Coronavirus (SARS-CoV-2) Using Molecular Docking Approach. ChemRxiv (Preprints): DOI: 10.26434/chemrxiv.12110214.

McBride, R., van Zyl, M. \& Fielding, B. C. (2014). The coronavirus nucleocapsid is a multifunctional protein. Viruses 6: 2991-3018. DOI: 10.3390/v6082991

Mielech, A. M., Chen, Y., Mesecar, A. D. \& Baker, S. C. (2014). Nidovirus papain-like proteases: multifunctional enzymes with protease, deubiquitinating and delSGylating activities. Virus Res 194: 184-190. DOI: 10.1016/j.virusres.2014.01.025

Pettersen, E. F., Goddard, T. D., Huang, C. C., Couch, G. S., Greenblatt, D. M., Meng, E. C. \& Ferrin, T. E. (2004). UCSF Chimera--a visualization system for exploratory research and analysis. J Comput Chem 25: 1605-1612. DOI: 10.1002/jcc.20084

Phillips, J. M., Gallagher, T. \& Weiss, S. R. (2017). Neurovirulent Murine Coronavirus JHM.SD Uses Cellular Zinc Metalloproteases for Virus Entry and Cell-Cell Fusion. Journal of Virology 91: e01564-01516. DOI: 10.1128/JVI.01564-16

Posthuma, C. C., Nedialkova, D. D., Zevenhoven-Dobbe, J. C., Blokhuis, J. H., Gorbalenya, A. E. \& Snijder, E. J. (2006). Site-directed mutagenesis of the Nidovirus replicative endoribonuclease NendoU exerts pleiotropic effects on the arterivirus life cycle. J Virol 80: 1653-1661. DOI: 10.1128/JVI.80.4.1653-1661.2006

Prajapat, M., Sarma, P., Shekhar, N., Avti, P., Sinha, S., Kaur, H., Kumar, S., Bhattacharyya, A., Kumar, H., Bansal, S. \& Medhi, B. (2020). Drug targets for corona virus: A systematic review. Indian journal of pharmacology 52: 56-65. DOI: 10.4103/ijp.IJP_115_20

Sturtz, F. G. (1998). Antimurine retroviral effect of doxycycline. Methods Find Exp Clin Pharmacol 20: 643-647. DOI: $10.1358 / \mathrm{mf} .1998 .20 .8 .487489$

Sutton, G., Fry, E., Carter, L., Sainsbury, S., Walter, T., Nettleship, J., Berrow, N., Owens, R., Gilbert, R., Davidson, A., Siddell, S., Poon, L. L., Diprose, J., Alderton, D., Walsh, M., Grimes, J. M. \& Stuart, D. I. (2004). The nsp9 replicase protein of SARS-coronavirus, structure and functional insights. Structure 12: 341-353. DOI: 10.1016/j.str.2004.01.016

Tay, M. Y., Fraser, J. E., Chan, W. K., Moreland, N. J., Rathore, A. P., Wang, C., Vasudevan, S. G. \& Jans, D. A. (2013). Nuclear localization of dengue virus (DENV) 1-4 non-structural protein 5; protection against all 4 DENV serotypes by the inhibitor Ivermectin. Antiviral Res 99: 301-306. DOI: 10.1016/j.antiviral.2013.06.002

van Aalten, D. M. F., Bywater, R., Findlay, J. B. C., Hendlich, M., Hooft, R. W. W. \& Vriend, G. (1996). PRODRG, a program for generating molecular topologies and unique molecular descriptors from coordinates of small molecules. Journal of Computer-Aided Molecular Design 10: 255262. https://doi.org/10.1007/BF00355047 
Wang, Q., Zhang, Y., Wu, L., Niu, S., Song, C., Zhang, Z., Lu, G., Qiao, C., Hu, Y., Yuen, K.-Y., Wang, Q., Zhou, H., Yan, J. \& Qi, J. (2020). Structural and Functional Basis of SARS-CoV-2 Entry by Using Human ACE2. Cell 181(4) 894-904.e9. https://doi.org/10.1016/j.cell.2020.03.045

Wu, C., Liu, Y., Yang, Y., Zhang, P., Zhong, W., Wang, Y., Wang, Q., Xu, Y., Li, M., Li, X., Zheng, M., Chen, L. \& Li, H. (2020). Analysis of therapeutic targets for SARS-CoV-2 and discovery of potential drugs by computational methods. Acta Pharmaceutica Sinica B.10(5):766-788. doi: 10.1016/j.apsb.2020.02.008.

Yang, J.-M., Chen, Y.-F., Tu, Y.-Y., Yen, K.-R. \& Yang, Y.-L. (2007). Combinatorial Computational Approaches to Identify Tetracycline Derivatives as Flavivirus Inhibitors. PLOS ONE 2: e428. DOI: 10.1371/journal.pone.0000428

Yang, S. N. Y., Atkinson, S. C., Wang, C., Lee, A., Bogoyevitch, M. A., Borg, N. A. \& Jans, D. A. (2020). The broad spectrum antiviral ivermectin targets the host nuclear transport importin $\alpha / \beta 1$ heterodimer. Antiviral Res 177: 3. DOI:10.1016/j.antiviral.2020.104760

Zhang, H., Penninger, J. M., Li, Y., Zhong, N. \& Slutsky, A. S. (2020). Angiotensin-converting enzyme 2 (ACE2) as a SARS-CoV-2 receptor: molecular mechanisms and potential therapeutic target. Intensive Care Medicine 46: 586-590. DOI:10.1007/s00134-020-05985-9

Zhou, Y., Hou, Y., Shen, J., Huang, Y., Martin, W. \& Cheng, F. (2020). Network-based drug repurposing for novel coronavirus 2019-nCoV/SARS-CoV-2. Cell Discovery 6: 14. DOI: 10.1038/s41421-0200153-3 